\title{
Neuromuscular Scoliosis: Comorbidities and Complications
}

\author{
Karen Andrea Weissmann ${ }^{1,2}$, Virginie Lafage ${ }^{3}$, Carlos Barrios Pitaque ${ }^{1}$, Renaud Lafage ${ }^{3}$, \\ Carlos M. Huaiquilaf ${ }^{4}$, Bryan $\mathrm{Ang}^{3}$, Ronald G. Schulz ${ }^{2}$ \\ ${ }^{I}$ Escuela de Doctorado, Universidad Católica de Valencia San Vicente Mártir, Valencia, Spain \\ ${ }^{2}$ Departamento de Ortopedia y Traumatología, Universidad de Chile, Santiago, Chile \\ ${ }^{3}$ Department of Surgery, Hospital for Special Surgery, New York, NY, USA \\ ${ }^{4}$ Hospital Exequiel Gonzalez Cortés, Redsalud, Santiago, Chile
}

Study Design: Single-center, retrospective cohort study conducted from 2013 to 2017.

Purpose: To determine the risk factors for surgical complications in neuromuscular scoliosis based on known patient comorbidities.

Overview of Literature: The concept of neuromuscular scoliosis includes a wide variety of pathologies affecting the neuromuscular system. Complications are numerous and are often difficult to predict.

Methods: A retrospective analysis of a single-center database was conducted from 2013 to 2017. Inclusion criteria were patients aged $<25$ years, diagnosis of neuromuscular scoliosis, and history of posterior fusion deformity surgery. A total of 64 patients (mean age, 15 years; $63 \%$ females) were included in this study. Clinical, radiological, and laboratory parameters in the preoperative, intraoperative, and postoperative settings were analyzed. Univariate analysis was performed using Student $t$-test for continuous variables, and a chi-square test was used for noncontinuous variables. Multivariate analysis was performed to identify predictors of major, mechanical, and total complications.

Results: Complications were found in $44 \%$ of patients, with $46.9 \%$ consisting of major complications, and $84.4 \%$ being early complications. Univariate analysis revealed that the presence of perinatal comorbidities, independent of other comorbidities, increased the risk for complications $(p=0.029$ ). Preoperative hypoglycemia, high number of instrumented levels, longer surgical time, use of an all-screw construct, lower preoperative pelvic obliquity, postoperative lower kyphosis, high thoracic spinopelvic angle (as measured by T9 spino-pelvic inclination), absence of deep drain, and use of superficial drain were associated with postoperative complications (all $p<0.05$ ). Logistic regression demonstrated that comorbidities, longer surgical time, hypoglycemia, and absence of deep drains are predictors of complications. Independent variables that predicted major complications were the number of levels fused, postoperative kyphosis ( $p=0.025$; odds ratio [OR], 1.074), and high screw density ( $p=0.014 ; 0 \mathrm{R}, 4.380)$.

Conclusions: Complications in neuromuscular scoliosis are increased by comorbidities, long surgical time, and inadequate correction. Preventative measures to decrease these complications include appropriate preoperative patient preparation and surgical planning.

Keywords: Scoliosis; Complications; Comorbidity; Perinatal

Received May 24, 2020; Revised Aug 30, 2020; Accepted Aug 30, 2020

Corresponding author: Karen Andrea Weissmann

Departamento de Ortopedia y Traumatología, Universidad de Chile, Area Sur. Gran Avenida Jose Miguel Carrera, 3100, San Miguel, Región Metropolitana, Chile

Tel: +56-9-84191581, Fax: +56-2-26720146, E-mail: karenw9@gmail.com, kweissmannm@uchile.cl 


\section{Introduction}

The concept of neuromuscular scoliosis includes a wide variety of pathologies associated with both the nervous and muscular systems [1]. With such a heterogeneous group of disorders, it is difficult to establish guidelines for preoperative planning, surgery, and postoperative treatment. Although patients are evaluated individually, identifying the shared characteristics can be valuable for treatment algorithms.

It is difficult to anticipate the complications in neuromuscular scoliosis surgery, with rates being reported from $6.3 \%$ to $50.0 \%$ [2-14]. The Scoliosis Research Society morbidity and mortality database for neuromuscular scoliosis reported a rate of $13 \%-16 \%$ between 2004 and 2007. This rate dramatically decreased to $4 \%$ between 2012 and 2015, and the authors primarily attributed this reduction to a two-fold decrease in infection rate.

Over the past 20 years, there have been insufficient, quality studies reporting the associations between neuromuscular scoliosis complications and controllable parameters, including ambulatory status, diagnosis of myelomeningocele, curve magnitude severity, instrumentation type, estimated blood loss, and pulmonary comorbidities [2-7,9-13,15] (Table 1). None of the published reports addressed the potential impact of perinatal comorbidities.

The Barker hypothesis proposes transgenerational epigenetic inheritance these changes might occur in as early as one generation [16]. Later life outcomes such as lower test scores, psychiatric diseases, and epilepsy have been associated with perinatal circumstances [16]. We hypothesized that epigenetic inheritance and perinatal

Table 1. Articles that studied the correlation between known parameters and complications in neuromuscular scoliosis

\begin{tabular}{|c|c|c|c|}
\hline Variable & No. of studies & Reference & Our study \\
\hline \multicolumn{4}{|l|}{ Demographics } \\
\hline Age $>13 y r$ & 2 & {$[9,12]$} & No, low Risser yes \\
\hline Nonwhite race & 1 & {$[7]$} & Not analyzed \\
\hline Family income & 1 & {$[7]$} & No, same results public/private hospital \\
\hline \multicolumn{4}{|l|}{ Comorbidities } \\
\hline$\geq 1$ known comorbidity & 2 & {$[7,9]$} & Yes \\
\hline Pulmonary comorbidity & 3 & {$[2,4,12]$} & No \\
\hline Known diagnosis: myelomeningocele or Duchenne & 4 & {$[2,9,10,15]$} & Yes \\
\hline Weight under $5 \%$ or over $95 \%$ & 2 & {$[2,9]$} & No \\
\hline Epilepsy & 1 & {$[2]$} & No \\
\hline Previous surgery & 1 & {$[2]$} & No \\
\hline Non-ambulatory & 4 & {$[2,9,11,13]$} & Yes \\
\hline \multicolumn{4}{|l|}{ Curves characteristics } \\
\hline Curve magnitude & 4 & {$[3,9,11,12]$} & No \\
\hline Curve rigidity & 1 & {$[3]$} & No \\
\hline Pelvic obliquity & 2 & {$[2,9]$} & No \\
\hline \multicolumn{4}{|l|}{ Surgery related } \\
\hline No. of levels instrumented & 1 & {$[7]$} & Yes \\
\hline One versus double staged surgery & 1 & {$[15]$} & No \\
\hline Type of instrumentation & 4 & {$[5,6,9,10]$} & No \\
\hline Osteotomies & 1 & {$[6]$} & No \\
\hline Surgical time & 2 & {$[2,9]$} & Yes \\
\hline Blood loss & 3 & {$[2,7,9]$} & Yes \\
\hline Inadequate reduction (LL or PO) & 1 & [2] & Yes, for major complications \\
\hline
\end{tabular}

LL, lumbar lordosis; PO, pelvic obliquity. 
comorbidities could potentially be related to complications in neuromuscular scoliosis surgery. Therefore, the present study was conducted to investigate the association between perinatal comorbidities, preoperative evaluation, surgical parameters, and postoperative complications in neuromuscular scoliosis.

\section{Materials and Methods}

This study was approved by the ethics committee for evaluation (approval no., 383/2019). Informed consent was obtained from all individual participants included in the study. A single-center retrospective analysis was conducted using the data of patients operated between August 2013 and October 2017. Inclusion criteria were patients aged $<25$ years, a diagnosis of neuromuscular scoliosis, and a past surgical history of a posterior spinal fusion. Exclusion criteria were history of revision spine surgery or use of growthguided systems. Data were collected from patient charts.

\section{Preoperative evaluation}

All patients underwent the same preoperative evaluation protocol consisting of a cardiac, pulmonary, psychological, and laboratory assessment. Data pertaining to perinatal morbidity were collected, including pregnancy characteristics, type of delivery, gestational weeks at birth, maternal diseases during pregnancy, birth weight, and birth size.

The following parameters concerning the current physical condition were evaluated: gait, preoperative and postoperative height and weight, comorbidities, previous surgeries, sports practiced, presence of thelarche in girls and increase in testicular volume in boys, age at menarche in girls or voice change in boys, use of preoperative brace, allergies, and use of medications. All patients underwent preoperative laboratory testing, including hematocrit ( $\mathrm{Ht}$, expressed as \%), hemoglobin ( $\mathrm{Hb}, \mathrm{g} / \mathrm{dL})$, and platelet count (100,000 platelets/field); coagulation analysis comprising activated partial thromboplastin time (seconds) and prothrombin time (\%); and a biochemical analysis of blood urinary nitrogen and glucose (both in $\mathrm{mg} / \mathrm{dL}$ ).

Patients were evaluated by a pediatric cardiologist using a bidimensional echocardiogram to search for any malformations. Ejection fraction was measured in patients when an adequate acoustic window permitted it. When possible, spirometry with and without a $\beta_{2}$-sympathomimetic agent was performed to measure forced expiratory volume dur- ing the first second (FEV1). When spirometry was not possible due to mental or physical issues, thoracic anteroposterior and lateral $\mathrm{X}$-rays, along with clinical evaluation, were used for preoperative assessment. Other respiratory parameters analyzed in this study were the use of nocturnal external respiratory aids such as bilevel positive airway pressure (BiPAP) and the number of lower respiratory tract infections (LRTI) per year in the preoperative and postoperative period.

All patients underwent a complete urinalysis and culture test. The number of urinary tract infections (UTIs) per year was recorded. If the urine culture test result was positive or the patient had previous urine culture tests, the colonizing bacterial type was evaluated. If a culture had $\leq 80,000$ colonies/field, it was considered as colonization and was left untreated.

Neurological evaluation was also performed when possible. Patients with epilepsy were preoperatively administered escitalopram for 3 days to avoid seizures related to neuromonitoring unless they were already taking this drug. The number of preoperative and postoperative seizures and the number and type of anticonvulsants taken were also recorded.

A psychological evaluation was performed on all complying patients to identify situations that would be contraindicated for surgery and to provide support and recommendations for families and patients. In general, three sessions were performed, including two before surgery and one postoperatively.

Preoperatively, an anesthesiologist evaluated all patients 1 month before surgery and classified the patients according to the American Society of Anesthesiologists (ASA) physical status classification [17]. Preoperative temperature $\left({ }^{\circ} \mathrm{C}\right)$, blood pressure $(\mathrm{mm} \mathrm{Hg})$, heart rate $(\mathrm{bpm})$, and oxygen saturation (\%) were also recorded.

Imaging evaluation was performed preoperatively, immediately postoperatively, and at 2-year follow-up and included full-length anteroposterior and lateral radiographs as well as preoperative bending and traction films to evaluate the curve flexibility. Radiographic parameters of interest included skeletal maturity assessed using the Risser scale, curve magnitude (Cobb angles), apical vertebral rotation assessed using the Raimondi method, pelvic obliquity (PO), cervical lordosis, thoracic kyphosis (TK), lumbar lordosis, pelvic incidence, sacral slope, pelvic tilt, sagittal vertical axis, T1 spino-pelvic inclincation, and T9 spino-pelvic inclination (T9SPI) [18-20]. All measure- 
ments were performed in Surgimap ver. 2.2.14.1 (Nemaris Inc., New York, NY, USA).

Details regarding the following intraoperative parameters were collected: blood $\mathrm{pH}, \mathrm{PCO}_{2}$ (partial pressure of carbon dioxide, $\mathrm{mmH}_{2} \mathrm{O}$ ), $\mathrm{PO}_{2}$ (partial pressure of oxygen, $\mathrm{mmH}_{2} \mathrm{O}$ ), $\mathrm{Ht} / \mathrm{Hb}$, screw density, type of rod material, type of derotation technique, osteotomies, surgical time (from skin incision to the placement of the dressing), intraoperative bleeding, number of red blood cells (RBCs), plasma and platelet transfusions, and the use type and dosage of vasoactive drugs. Neuromonitoring data were analyzed regarding baselines, endlines, and any alerts.

Postoperative parameters evaluated in this study included length of stay (LOS) at each unit, admission to the intensive care unit (ICU) with or without mechanical ventilation, airway status (intubated or extubated), Ht, $\mathrm{Hb}$, platelets, and $\mathrm{C}$-reactive protein at postoperative days 1,3 , and 7. Postoperative transfusions were also evaluated. Postoperative bleeding was evaluated by measuring the drain output and the number of drain days.

Early complications were defined as complications that occurred in the perioperative period up to 12 months postoperatively. Complications classified after this time period were considered as late complications. Major complications were defined as those that pose a risk to the patient's life, and mechanical complications were any of those related to the osteosynthesis material.

\section{Statistical analysis}

Univariate analysis was conducted to compare patients who sustained a complication versus those who did not using a chi-square or a two-tailed Student $t$-test. Similar analyses were conducted for major and mechanical complications. Statistically significant parameters identified in the univariate analysis were then used in a binary logistic regression to examine the variables with the largest impact associated with the likelihood of developing complications. Statistical analysis was conducted using IBM SPSS ver. 19.0 (IBM Corp., Armonk, NY, USA), and the level of significance was set at 0.05 .

\section{Results}

\section{Demographics}

A total of 64 consecutive patients were included in this
Table 2. Primary diagnosis

\begin{tabular}{|c|c|}
\hline Diagnosis & No. of frequency \\
\hline \multicolumn{2}{|l|}{ Neuropathics } \\
\hline \multicolumn{2}{|l|}{ First motoneuron } \\
\hline Cerebral palsy & 20 \\
\hline Chiari type I & 4 \\
\hline Siringomyelia & 3 \\
\hline Intradural tumors & 2 \\
\hline Spinocerebelar ataxia & 1 \\
\hline Spinal cord trauma & 2 \\
\hline \multicolumn{2}{|l|}{ Second motoneuron } \\
\hline Charcot-Marie-Tooth & 1 \\
\hline Spinal atrophie type II & 3 \\
\hline Congenital hypothonia & 6 \\
\hline \multicolumn{2}{|l|}{ Mixed } \\
\hline Tetherd cord & 2 \\
\hline Myelomeningocele & 8 \\
\hline \multicolumn{2}{|l|}{ Myopathic } \\
\hline Duchenne syndrome & 1 \\
\hline Central core disease & 1 \\
\hline \multicolumn{2}{|l|}{ Colagenopathies } \\
\hline Marfan syndrome & 3 \\
\hline Ehler-Danlos syndrome & 7 \\
\hline
\end{tabular}

study, with a follow-up time of 24 months. The majority of cohort consisted of female patients (62.5\%), with a mean age at surgery of 15 years (range, 8-25 years). Motor neuron pathologies accounted for $50 \%$ of the cohort, with cerebral palsy being the most common diagnosis in this group (Table 2 ).

In total, $51.6 \%$ of the patients were treated in the public system, and $48.4 \%$ were treated in the private system by the same surgical team and primary surgeon. Preoperative weight (mean, $45.49 \pm 44.48 \mathrm{~kg}$ ) significantly increased (mean, $51.45 \pm 15.17 \mathrm{~kg}$ ) at 2-year follow-up ( $p=0.030$ ), as did mean height (preoperative, $135.09 \pm 44.49 \mathrm{~cm}$; 2-year follow-up, $148.74 \pm 33.81 \mathrm{~cm} ; p<0.001)$.

\section{Complications}

The mean complication rate was $44.4 \%$ (28 patients with 32 complications), and early complications accounted for $84.4 \%$ of all complications (Table 3 ). Among patients with complications, $7.7 \%$ had a surgical site infection $(3.1 \%$ 


\begin{tabular}{lc|}
\hline Table 3. Complications & \\
\hline Complication & Rate (\%) \\
\hline Superficial infection & 3.13 \\
\hline Deep infection & 4.7 \\
\hline Occipital pressure sore & 1.6 \\
\hline Bilateral pneumonia & 4.7 \\
\hline Minor pneumothorax/atelectasis & 4.7 \\
\hline Varicella & 1.6 \\
\hline Intraoperative screw pullout repositioned during same surgery & 1.6 \\
\hline Unidentified fever for 2 days & 4.7 \\
\hline Incidental durotomy & 3.13 \\
\hline Hypovolemic shock & 4.7 \\
\hline Tongue biting & 1.6 \\
\hline Postoperative convulsions & 3.13 \\
\hline Paraparesis (complete recovery 3 weeks) & 1.6 \\
\hline Postoperative paralytic ileum & 1.6 \\
\hline Late complications & 3.13 \\
\hline Proximal screw pullout due to proximal junctional kyphosis & 1.6 \\
\hline Pedicle fracture due to traumatic accident & 3.13 \\
\hline Nonunion & \\
\hline
\end{tabular}

superficial and $4.7 \%$ deep), and $4.7 \%$ were diagnosed with pneumonia. Vancomycin and amikacin were the most commonly used antibiotics in infected patients. Among patients with deep infection, one was diagnosed with varicella and received extended treatment with acyclovir. Two incidental durotomies were repaired during surgery without further complications.

Major complications were found in $49.9 \%$ of patients, with all complications occurring in the early period (i.e., before 1 year). Among these patients, three recuperated from hypovolemic shock, $9.4 \%$ were due to respire, and two patients had status epilepticus. There were no deaths in this series. Late complications were all mechanical in nature and observed in $7.8 \%$ of patients; two of these were nonunions requiring revision, and both were myelomeningoceles with sacroiliac nonunion and underwent anterior lumbar interbody fusion with good results.

In total, $63 \%$ of patients had normal baselines in the four extremities, and $8 \%$ had only readable somatosensory evoked potential (SSEP) and motor evoked potential (MEP) in the upper extremities. All other patients had either absent, altered, or incomplete lines in the upper and lower extremities. A total of 10 patients had intraoperative neuromonitoring alerts, of which four were perfusion-related and spontaneously recuperated upon increasing the blood pressure, whereas two were minor alerts during detethering that recuperated immediately after the completion of this part of procedure. Among the remaining four alerts, three patients had a decrease in MEP in one lower extremity, primarily anterior tibialis and adductor hallucis longus with no clinical postoperative consequence, and one patient with Ehlers-Danlos syndrome had a complete loss of lower extremity SSEP and MEP. Partial improvement was observed after activation of the intraoperative protocol ( $<50 \%$ at final follow-up), but the patient woke up paraparetic (M2 right lower extremity and M3 left lower extremity), with complete recovery at 3 weeks.

\section{Clinical evaluation}

\section{1) Perinatal characteristics}

In total, $78.5 \%$ of the mothers had normal pregnancies, $54.8 \%$ of deliveries where eutocic, $41.9 \%$ were C-sections, and 3.2\% required the use of forceps. Delivery occurred at a mean of 37.9 weeks (range, 26-41 weeks), with a mean birth weight of 3,186 g (range, 1,040-4,280 g) and a mean size of $48.2 \mathrm{~cm}$ (range, 32-54 cm). Furthermore, 33.3\% of patients had no perinatal pathologies. A prenatal diagnosis of pathology involving the central nervous system (myelomeningocele) was found in $14 \%$ of mothers. The most common perinatal conditions besides morphological alterations of the spinal canal (seven patients) were genopathies, prematurity, and perinatal pneumonias.

\section{2) Comorbidities}

At least one comorbidity was found in $93.75 \%$ of patients, whereas $56 \%$ of them had three or more comorbidities, with a maximum of 10 comorbidities.

\section{3) Previous surgery}

In total, $62.5 \%$ of patients had undergone previous surgeries, $46.9 \%$ had undergone fewer than three surgeries, and $15.7 \%$ had undergone more than four surgeries.

\section{4) Current health}

A total of $73.8 \%$ of patients underwent surgery post menarche or voice change at a mean age of 12.8 years. Of those premenarchial patients, $17.7 \%$ had already undergone thelarche or displayed secondary sexual characteristics at a mean age of 10.27 years. No allergies were found in 
$70.8 \%$ of patients, whereas $18.5 \%$ were allergic to latex (all myelomeningocele cases), $4.6 \%$ were allergic to penicillin, and $3 \%$ had other allergies. There were no sports activities in $85.9 \%$ of patients, whereas $32.3 \%$ of them had undergone a previous treatment with a brace.

\section{5) Cardiac evaluation}

No cardiac alterations were found in $73.4 \%$ of patients. Among those with morphological findings, the majority were mild tricuspid insufficiencies. Ejection fraction could be measured in only 15/64 patients, with the mean value being $71.53 \% \pm 14.78 \%$. Two patients had life-threatening malformations, including one patient who had a severe aortic dilatation with mitral valve reflux and cava dilatation with Marfan syndrome that required evaluation by a cardiothoracic surgeon; however, no cardiac surgery was recommended at that point and posterior spine fusion was subsequently authorized. The other patient had cardiac insufficiency due to cor pulmonale secondary to a $144^{\circ}$ curve magnitude that required permanent digoxin to maintain cardiac function.

\section{6) Bronchopulmonary evaluation}

Standard spirometry evaluation could be completed in $29.7 \%$ of patients, with the mean FEV1 being $73.16 \% \pm 17.58 \%$. Preoperatively, $60 \%$ of patients had more than three episodes of LRTI a year, of whom $15.6 \%$ required domiciliary nocturnal BiPAP. Postoperatively, 8.1\% of patients presented with an LRTI, and the difference between the preoperative and postoperative number of LRTIs was significantly different $(p<0.000)$.

\section{7) Urological evaluation}

Recurrent lower UTIs before surgery were found in $21.5 \%$ of patients; $69 \%$ of these patients had three or fewer UTIs per year, whereas $31 \%$ had up to six UTIs per year. After surgery, the UTI prevalence was $12.5 \%$, with $75 \%$ of these patients having fewer than three UTIs per year and 25\% having up to five UTIs per year; this decrease was significantly different $(p=0.015)$. In particular, $77.77 \%$ of the identified bacteria were Gram-negative.

\section{8) Neurological evaluation}

Preoperatively, $15.6 \%$ of patients suffered from one to four seizures per month. Of these patients, $20 \%$ were under anticonvulsant treatment, with $54 \%$ being treated with valproic acid, and all of them were under multi-pharma- cological treatment. Postoperatively, 6.3\% of patients still experienced seizures but at a maximum rate of once per month $(p=0.007)$.

9) Psychological evaluation

Psychiatric evaluation was performed in $71.9 \%$ of patients, and no psychotic or personality disorders were diagnosed. Although all patients undergoing psychiatric evaluation exhibited anxiety symptoms, no surgery was suspended or rescheduled due to psychological pathology.

10) Anesthesia

In total, $54.7 \%$ of patients had ASA 2, and $20.3 \%$ had ASA 3. Antibiotic prophylaxis with cefazolin was implemented in $96.6 \%$ of patients, whereas $3.4 \%$ received clindamycin. Vasoactive drugs were required to maintain hemodynamic stability in $34.4 \%$ of patients, of which phenylephrine was used in $25 \%$ of patients, epinephrine or norepinephrine in $28 \%$, and $3 \%$ of them required the addition of dopamine.

11) Surgical technique

A total of 833 levels were instrumented. Titanium rods were used in $54.7 \%$ of patients, and CrCo rods were used in $40.6 \%$ of patients. In three patients, one rod of each material was placed. Screw placement was primarily a low-density screw technique (in $72.6 \%$ of patients). In $29.6 \%$ of patients, the instrumentation was performed to the pelvis. In $53.1 \%$ of patients, the coplanar technique was used for derotation, whereas apical rotation was used in $46.9 \%$ of patients. Osteotomies were performed in $40 \%$ of patients; $81 \%$ of these were classified as Schwab 2 [21]. Eight patients were simultaneously detethered, and no postoperative fistulas were formed. The mean surgical time was 217.4 \pm 90.7 minutes.

\section{2) Surgical correction}

A total of $69 \%$ of patients had Risser scale scores of III to $\mathrm{V}$. The mean preoperative maximum Cobb angle was $65.7^{\circ} \pm 25.5^{\circ}$, and by $11.2^{\circ} \pm 16.5^{\circ}$ and $18.9^{\circ} \pm 12.7^{\circ}$, respectively, on bending and traction films (both with $p<0.001$ ). The maximum Cobb angle was corrected to $33.9^{\circ} \pm 23.6^{\circ}$ $(p<0.001)$ postoperatively, with a slight loss of correction at 2 years $\left(3.2^{\circ} \pm 8.3^{\circ}, p=0.026\right)$. PO was also significantly corrected $(p=0.017)$ and maintained at 2 years. In the sagittal plane, TK was significantly decreased from $41.0^{\circ} \pm 28.5^{\circ}$ to $34.1^{\circ} \pm 17.4^{\circ}(p=0.015)$, with no further change at 2-year follow-up (Table 4 ). 


\section{3) Bleeding}

Operative laboratory parameters were normal in all patients, with a mean $\mathrm{Ht} / \mathrm{Hb}$ of $40.35 \% / 13.26 \mathrm{~g} / \mathrm{dL}$, which decreased to $30.88 \% / 10.51 \mathrm{~g} / \mathrm{dL}$ during the surgery and stabilized at $28.64 \% / 9.9 \mathrm{~g} / \mathrm{dL}$ in the postoperative period and $30.16 \% / 12.44 \mathrm{~g} / \mathrm{dL}$ at discharge (Table 5). Total blood

Table 4. Pre- and postoperative radiographic measurements

\begin{tabular}{|c|c|c|c|c|c|c|c|}
\hline Measurement & Preoperative & Postoperative & Correction & $p$-value & 2-yr FU & $\%$ Correction at 2-yr FU & $p$-value \\
\hline $\operatorname{Max} \operatorname{Cobb}\left({ }^{\circ}\right)$ & $65.7 \pm 25.5$ & $30.6 \pm 22.2$ & $-33.9 \pm 14.2$ & 0.000 & $33.9 \pm 23.6$ & $3.2 \pm 8.3$ & 0.026 \\
\hline Bending $\left({ }^{\circ}\right)$ & $56.1 \pm 28.2$ & - & $11.2 \pm 16.5$ & 0.000 & - & - & - \\
\hline Traction $\left({ }^{\circ}\right)$ & $50.91 \pm 25.9$ & - & $18.9 \pm 12.7$ & 0.000 & - & - & - \\
\hline Pelvic obliquity $\left({ }^{\circ}\right)$ & $12.34 \pm 16.88$ & $6.54 \pm 10.14$ & $-3.5 \pm 10.2$ & 0.017 & $6.3 \pm 8.7$ & $0.3 \pm 6.8$ & 0.772 \\
\hline Apical vertebral rotation $\left({ }^{\circ}\right)$ & $18.9 \pm 12.5$ & $10.5 \pm 11.1$ & $-7.5 \pm 13.1$ & 0.000 & $11.2 \pm 10.7$ & $1.1 \pm 8.7$ & 0.461 \\
\hline Lordosis cervical $\left(\left(^{\circ}\right)\right.$ & $13.74 \pm 13.5$ & $17.6 \pm 17.0$ & $1.7 \pm 15.2$ & 0.405 & $14.5 \pm 13.6$ & $-5.2 \pm 16.3$ & 0.041 \\
\hline Thoracic kyphosis $\left({ }^{\circ}\right)$ & $41.0 \pm 28.5$ & $34.1 \pm 17.46$ & $8.4 \pm 24.5$ & 0.015 & $33.8 \pm 19.57$ & $0.9 \pm 9.3$ & 0.507 \\
\hline Lordosis lumbar $\left({ }^{\circ}\right)$ & $54.6 \pm 19.85$ & $51.89 \pm 15.2$ & $-3.9 \pm 15.28$ & 0.066 & $49.1 \pm 16.1$ & $-2.4 \pm 12.1$ & 0.190 \\
\hline Sacral slope $\left({ }^{\circ}\right)$ & $35.6 \pm 11.13$ & $35.0 \pm 10.67$ & $-2.1 \pm 6.7$ & 0.836 & $34.0 \pm 8.1$ & $0.5 \pm 6.1$ & 0.821 \\
\hline Pelvic incidence $\left({ }^{\circ}\right)$ & $46.1 \pm 21.56$ & $48.9 \pm 16.0$ & $-2.9 \pm 6.5$ & 0.495 & $47.3 \pm 14.8$ & $-1.0 \pm 6.2$ & 0.533 \\
\hline Pelvic tilt $\left({ }^{\circ}\right)$ & $13.2 \pm 11.62$ & $13.87 \pm 10.3$ & $-0.6 \pm 6.9$ & 0.760 & $14.0 \pm 11.6$ & $0.3 \pm 7.0$ & 0.750 \\
\hline Sagittal vertical axis $(\mathrm{mm})$ & $26.75 \pm 39.3$ & $28.8 \pm 46.5$ & $2.4 \pm 59.8$ & 0.766 & $34.0 \pm 39.8$ & $5.1 \pm 50.0$ & 0.493 \\
\hline $\operatorname{TgSPI}\left({ }^{\circ}\right)$ & $11.5 \pm 11.0$ & $8.3 \pm 8.8$ & $-2.9 \pm 12.2$ & 0.085 & $9.1 \pm 7.5$ & $-0.5 \pm 8.5$ & 0.702 \\
\hline $\operatorname{T} 1 \mathrm{SPI}\left({ }^{\circ}\right)$ & $2.0 \pm 6.8$ & $3.4 \pm 7.0$ & $1.3 \pm 7.9$ & 0.206 & $5.1 \pm 5.1$ & $1.7 \pm 8.8$ & 0.303 \\
\hline
\end{tabular}

Values are presented as mean \pm standard deviation. Bold type is considered statistically significant. FU, follow-up; SPI, spino-pelvic inclination.

Table 5. Laboratory parameters pre- to postoperative

\begin{tabular}{|c|c|c|c|c|}
\hline Variable & Preoperative & Intraoperative & Postoperative & At discharge \\
\hline Hematocrit (\%) & $40.35 \pm 3.49$ & $30.88 \pm 4.85$ & $28.64 \pm 4.8$ & $30.16 \pm 4.11$ \\
\hline Hemoglobin (g/dL) & $13.26 \pm 1.4$ & $10.51 \pm 1.58$ & $9.9 \pm 1.74$ & $12.44 \pm 12.52$ \\
\hline Platelets (thousand/field) & $273.610 \pm 72.23$ & - & - & - \\
\hline Activated partial thromboplastin time (sec) & $30.39 \pm 3.57$ & - & - & - \\
\hline Prothrombin (\%) & $84.33 \pm 26.95$ & - & - & - \\
\hline Glucose (mg/dL) & $87.61 \pm 8.35$ & - & - & - \\
\hline Blood urinary nitrogen (mg/dL) & $14.43 \pm 4.72$ & - & - & - \\
\hline Temperature $\left({ }^{\circ} \mathrm{C}\right)$ & $36.06 \pm 1.37$ & - & $35.9 \pm 0.99$ & - \\
\hline Medial arterial pressure $(\mathrm{mm} \mathrm{Hg})$ & $110.31 / 63.34$ & - & $102.61 / 57.07$ & - \\
\hline Heart rate (bpm) & $84.74 \pm 18.72$ & - & - & - \\
\hline Saturation $\mathrm{O}_{2}(\%)$ & $98.26 \pm 1.48$ & - & - & - \\
\hline $\mathrm{PH}$ & - & $7.35 \pm 0.09$ & - & - \\
\hline $\mathrm{PCO}_{2}\left(\mathrm{mmH}_{2} \mathrm{O}\right)$ & - & $41.67 \pm 7.9$ & - & - \\
\hline $\mathrm{PO}_{2}\left(\mathrm{mmH}_{2} \mathrm{O}\right)$ & - & $9.63 \pm 66.70$ & - & - \\
\hline C-reactive protein day 1 (mg/L) & - & - & $9.0 \pm 7.5$ & - \\
\hline C-reactive protein day 3 (mg/L) & - & - & $115.65 \pm 88.96$ & - \\
\hline C-reactive protein day 7 (mg/L) & - & - & $81.90 \pm 54.21$ & - \\
\hline
\end{tabular}

Values are presented as mean \pm standard deviation or number.

$\mathrm{PCO}_{2}$, partial pressure of carbon dioxide; $\mathrm{PO}_{2}$, partial pressure of oxygen. 


\begin{tabular}{lcr} 
Table 6. Bleeding & & \\
Variable & Mean \pm standard deviation & Min-max \\
\hline Intraoperative bleeding $(\mathrm{mL})$ & $938.89 \pm 668.016$ & $150-4,000$ \\
\hline Total drain debit $(\mathrm{mL})$ & $405.36 \pm 387.47$ & $0-1,875$ \\
\hline Total blood loss $(\mathrm{mL})$ & $1,423.21 \pm 866.35$ & $175-4,425$ \\
\hline Intraoperative transfusions & $1.09 \pm 0.937$ & $0-1$ \\
\hline Postoperative transfusions & $0.43 \pm 0.47$ & $0-3$ \\
\hline
\end{tabular}

loss volume, including drain output, was 1,423.21 \pm 866.35 $\mathrm{mL}$ (Table 6). In $54.6 \%$ of patients, intravenous tranexamic acid (TXA) was used, and in $82.8 \%$ of patients, topical TXA was used (Table 7). Intraoperatively, 44 patients were transfused with RBCs, nine patients were transfused with platelets, and 14 patients were transfused with plasma. Postoperatively, 23 patients required RBC transfusions, and none required additional plasma or platelets (Table 6). Superficial drains were used in 48 patients, deep drains were used in 12 patients, and no drain was used in 16 patients. The mean number of days of drain use was 3.19 with a maximum of 7 days.

\section{4) Hospitalization}

A total of $26.6 \%$ of patients were sent to the ICU while intubated, of whom eight were mechanically ventilated after detethering to avoid Valsalva maneuver. Another $32.3 \%$ of patients were sent directly to the intermediate care unit. The mean length of hospital stay was 3.5 days.

\section{Risk analysis}

Patients with and without overall complications were compared using univariate analysis based on all the abovedescribed preoperative, intraoperative, and postoperative parameters (Table 7). In terms of demographic data, there were no significant differences between patients with or without complications. The number of comorbidities was significantly higher in patients with complications (mean of 4.0 versus $2.5, p=0.009$ ).

Patients with complications were more likely to have a history of perinatal comorbidities $(p=0.029)$, independent of other comorbidities; no other perinatal parameters were statistically significant. The laboratory findings revealed that patients with complications had a significantly lower preoperative glycemia ( 84.7 versus $89.9 \mathrm{~g} / \mathrm{dL}$, $p=0.016$ ).
Intraoperatively, patients with more number of complications had more levels instrumented $(p=0.044)$, longer surgical time $(p=0.012)$, presence of an all-screw construct $(p=0.010)$, absence of a deep drain $(p=0.003)$, or use of superficial drain $(p=0.021)$. Osteosynthesis material, simultaneous detethering, and use of osteotomies were not significant. Total bleeding was almost significant with a p-value of 0.065 .

Patients with complications had a smaller preoperative PO $(p=0.050)$, smaller postoperative kyphosis $(p=0.035)$, larger flattening of TK $\left(10.8^{\circ} \pm 27.7^{\circ}\right.$ versus $\left.6.3^{\circ} \pm 21.6^{\circ}\right)$, and higher postoperative T9SPI ( $p=0.043$ ) (Table 8).

In the multivariate logistic regression analysis, presence of comorbidities ( $p=0.026$; odds ratio [OR], 1.423), lower preoperative glycemia ( $p=0.003$; OR, 0.865 ), longer surgical time ( $p=0.020$; OR, 1.007), and absence of a deep drain ( $p=0.017$; OR, 0.124 ) were identified as independent predictors for the presence of complications. For major complications, the independent predictors were the number of levels fused ( $p=0.046$; OR, 1.311) and lower postoperative kyphosis ( $p=0.025$; OR, 1.074). Finally, a high screw density ( $p=0.014$; OR, 4.380 ) was the only independent predictor of mechanical complications.

\section{Discussion}

The best defense against complications is to prevent them. In a study of 2,154 patients with neuromuscular scoliosis, the overall complication rate was $33.1 \%-40.1 \%$, an unacceptable value for any surgical procedure $[7,14]$, indicating that it is necessary to assess patients in advance to obtain all pertinent information that might impact a complication. Patient history must begin before the patient is even born, including the mother's pregnancy and birthing circumstances. We did not find any study analyzing the association between perinatal characteristics and complications; however, our findings support the importance of perinatal pathologies in the incidence of surgical complications in neuromuscular scoliosis. In a retrospective study that evaluated the cause of complications in 7,252 children with neuromuscular scoliosis aged $>5$ years, $59 \%$ of them were found to have more than four comorbidities, which correlated with a higher incidence of surgical complications [22]. Similarly, in our study, the presence of four or more comorbidities served as an independent predictor of complications.

Conditions strongly associated with longer hospital- 
Table 7. Risk assessment for complications

\begin{tabular}{|c|c|c|c|c|}
\hline Variable & Category & With complication & No complication & $p$-value \\
\hline \multirow[t]{16}{*}{ Demographic } & Age (yr) & $14.86 \pm 2.44$ & $15.44 \pm 4.36$ & 0.498 \\
\hline & Sex (male:female) & $11: 14$ & $25: 14$ & 0.093 \\
\hline & Preop weight $(\mathrm{kg})$ & $45.06 \pm 14.88$ & $45.93 \pm 16.90$ & 0.840 \\
\hline & Preop size $(\mathrm{cm})$ & $148.8 \pm 15.4$ & $146.7 \pm 19.14$ & 0.730 \\
\hline & Hospital (public/private) & $18 / 10$ & $15 / 21$ & 0.369 \\
\hline & No. of comorbidities & $3.96 \pm 2.5$ & $2.47 \pm 1.6$ & 0.009 \\
\hline & Previous surgeries (yes/no) & $19 / 9$ & $21 / 15$ & 0.302 \\
\hline & Thelarche age (yr) & $9.75 \pm 2.5$ & $10.57 \pm 1.13$ & 0.464 \\
\hline & Menarche age (yr) & $12.65 \pm 1.7$ & $12.93 \pm 1.7$ & 0.589 \\
\hline & Preop brace (yes/no) & $12 / 16$ & $9 / 27$ & 0.107 \\
\hline & Allergies (yes/no) & $8 / 20$ & $10 / 26$ & 0.320 \\
\hline & Ambulatory status (yes/no) & $16 / 12$ & $24 / 11$ & 0.250 \\
\hline & No. of seizures $(/ \mathrm{mo})$ & $0.5 \pm 1.2$ & $0.28 \pm 0.9$ & 0.390 \\
\hline & No. of UTI (/yr) & $0.9 \pm 1.5$ & $0.3 \pm 1.1$ & 0.095 \\
\hline & No. of LRTI (/yr) & $1.0 \pm 1.7$ & $0.67 \pm 1.5$ & 0.458 \\
\hline & No. of patients (ASA I/II/III) & $4 / 17 / 7$ & $12 / 18 / 6$ & 0.207 \\
\hline \multirow[t]{6}{*}{ Perinatal } & Birth weight (kg) & $3.176 \pm 739$ & $3194 \pm 596$ & 0.914 \\
\hline & Birth size $(\mathrm{cm})$ & $48.23 \pm 4.8$ & $48.07 \pm 3.83$ & 0.873 \\
\hline & Delivery (wk) & $37.62 \pm 2.9$ & $38.17 \pm 2.6$ & 0.436 \\
\hline & Pathological pregnancy (normal/pathological) & $6 / 31$ & $6 / 20$ & 0.273 \\
\hline & Delivery (eutocic/c-section) & $16 / 9$ & 18/17 & 0.609 \\
\hline & Perintal pathology (no/yes) & $14 / 13$ & $28 / 8$ & 0.029 \\
\hline \multirow[t]{4}{*}{ Laboratory } & Preop glicemia (mg/dL) & $84.7 \pm 8.2$ & $89.9 \pm 7.9$ & 0.016 \\
\hline & Preop blood bitrogen (mg/dL) & $15.75 \pm 5.0$ & $13.4 \pm 4.3$ & 0.055 \\
\hline & Hematocrit preop (\%) & $40.4 \pm 4.2$ & $40.3 \pm 4.2$ & $0.973 / 0.908$ \\
\hline & Hemoglobin preop (g/dL) & $13.3 \pm 1.8$ & $13.27 \pm 1.1$ & - \\
\hline \multirow[t]{15}{*}{ Surgical } & Rod material (titanium/CrCo/both) & $19 / 7 / 2$ & $16 / 19 / 1$ & 0.074 \\
\hline & All screws/low density & $23 / 5$ & $24 / 12$ & 0.010 \\
\hline & No. of instrumented levels & $13.93 \pm 2.9$ & $12.31 \pm 3.3$ & 0.044 \\
\hline & LIV to pelvis (yes/no) & $8 / 28$ & $11 / 17$ & 0.458 \\
\hline & Surgical technique (VCA/AD) & 13/15 & $21 / 15$ & 0.244 \\
\hline & Osteotomies Schawb (1-6) & $0 / 11 / 2 / 0 / 0 / 0$ & $0 / 11 / 2 / 1 / 0 / 0$ & 0.592 \\
\hline & Simultaneous dethethering (yes/no) & $4 / 24$ & $4 / 32$ & 0.495 \\
\hline & Surgical time (min) & $245.0 \pm 80.2$ & $189.7 \pm 86.7$ & 0.012 \\
\hline & Bleeding $(\mathrm{mL})$ & $1,112 \pm 865$ & $800 \pm 418$ & 0.065 \\
\hline & Use of intravenous TXA (yes/no) & $17 / 19$ & 18/10 & 0.134 \\
\hline & Use of topical TXA (yes/no) & $29 / 7$ & $24 / 4$ & 0.296 \\
\hline & No. of transfusions-red blood cells & $3 \pm 2.0$ & $1.75 \pm 1.2$ & 0.123 \\
\hline & No. of transfusions-platelet & $2.88 \pm 2.9$ & $3.7 \pm 3.1$ & 0.700 \\
\hline & Hematocrit (\%) & $27.6 \pm 5.7$ & $29.5 \pm 3.9$ & 0.170 \\
\hline & Hemoglobine (g/dL) & $9.5 \pm 2.0$ & $10.23 \pm 1.4$ & 0.092 \\
\hline
\end{tabular}


Table 7. Continued

\begin{tabular}{|c|c|c|c|c|}
\hline Variable & Category & With complication & No complication & $p$-value \\
\hline & $\mathrm{pH}$ & 7.3 & 7.3 & 0.578 \\
\hline & $\mathrm{PO}_{2}\left(\mathrm{mmH}_{2} \mathrm{O}\right)$ & 111.9 & 86.5 & 0.298 \\
\hline & $\mathrm{pCO}_{2}\left(\mathrm{mmH}_{2} \mathrm{O}\right)$ & 40.7 & 42.7 & 0.465 \\
\hline & Drain (superficial) & 17 & 11 & 0.021 \\
\hline & Drain (deep) & 10 & 18 & 0.003 \\
\hline & Intensive care unit & 4.65 & 2.29 & 0.178 \\
\hline & Intensive treatment unit & 3.16 & 1.26 & 0.108 \\
\hline & Medical-surgical unit & 6.77 & 3.55 & 0.094 \\
\hline & Mechanical ventilation (yes/no) & $7 / 18$ & $7 / 26$ & 0.578 \\
\hline
\end{tabular}

Values are presented as mean \pm standard deviation or number, unless otherwise stated. Bold type is considered statistically significant.

Preop, preoperative; Postop, postoperative; UTI, urinary tract infections; LRTI, lower respiratory tract infections; ASA, American Society of Anesthesiologists; LIV, lower instrumented vertebra; VCA, vertebral coplanar alignement; $\mathrm{AD}$, apical derotation; $\mathrm{TXA}$, tranexamic acid; $\mathrm{PCO}_{2}$, partial pressure of carbon dioxide; $\mathrm{PO}_{2}$, partial pressure of oxygen.

Table 8. Radiological parameters and their complication risk

\begin{tabular}{|c|c|c|c|c|c|c|}
\hline \multirow{2}{*}{ Variable } & \multicolumn{3}{|c|}{ Preoperative } & \multicolumn{3}{|c|}{ Postoperative } \\
\hline & With complications & Without complications & $p$-value & With complications & Without complications & $p$-value \\
\hline Risser (0-V) & $3 / 5 / 1 / 1 / 8 / 10$ & $3 / 2 / 6 / 3 / 11 / 11$ & 0.362 & - & - & - \\
\hline Curve magnitude $\left({ }^{\circ}\right)$ & $69.7 \pm 27.7$ & $62.3 \pm 23.3$ & 0.271 & $34.8 \pm 22.6$ & $26.9 \pm 21.7$ & 0.186 \\
\hline Apical vertebral rotation $\left({ }^{\circ}\right)$ & $19.7 \pm 14.3$ & $18.2 \pm 10.8$ & 0.668 & $12.3 \pm 12.4$ & $9.1 \pm 10.0$ & 0.301 \\
\hline Pelvic obliquity $\left({ }^{\circ}\right)$ & $7.27 \pm 8.4$ & $16.8 \pm 21.0$ & 0.050 & $8.4 \pm 12.5$ & $5.1 \pm 8.0$ & 0.220 \\
\hline Cervical lordosis $\left({ }^{\circ}\right)$ & $13.2 \pm 11.9$ & $14.2 \pm 14.9$ & 0.782 & $16.6 \pm 16.6$ & $18.4 \pm 17.4$ & 0.683 \\
\hline Thoracic kyphosis $\left({ }^{\circ}\right)$ & $40.9 \pm 31.8$ & $41.2 \pm 25.7$ & 0.976 & $28.9 \pm 15.2$ & $38.2 \pm 18.1$ & 0.035 \\
\hline Lumbar lordosis $\left({ }^{\circ}\right)$ & $53.4 \pm 22.8$ & $55.7 \pm 17.0$ & 0.670 & $49.4 \pm 14.2$ & $53.8 \pm 15.8$ & 0.267 \\
\hline Sacral slope $\left({ }^{\circ}\right)$ & $37.4 \pm 11.0$ & $34.1 \pm 11.2$ & 0.265 & $36.1 \pm 11.8$ & $34.2 \pm 9.8$ & 0.491 \\
\hline Pelvic incidence $\left({ }^{\circ}\right)$ & $49.9 \pm 20.4$ & $43.16 \pm 22.3$ & 0.230 & $45.9 \pm 14.5$ & $48.4 \pm 15.1$ & 0.815 \\
\hline Pelvic tilt $\left({ }^{\circ}\right)$ & $16.04 \pm 11.8$ & $10.9 \pm 11.2$ & 0.085 & $13.6 \pm 13.4$ & $14.3 \pm 10.4$ & 0.565 \\
\hline Sagittal vertical axis (mm) & $28.1 \pm 29.9$ & $25.5 \pm 46.7$ & 0.812 & $43.6 \pm 32.2$ & $26.3 \pm 44.2$ & 0.364 \\
\hline T9 spino-pelvic inclination $\left({ }^{\circ}\right)$ & $10.4 \pm 11.6$ & $12.4 \pm 10.6$ & 0.505 & $10.4 \pm 6.8$ & $8.2 \pm 8.0$ & 0.043 \\
\hline T1 spino-pelvic inclination $\left({ }^{\circ}\right)$ & $0.9 \pm 7.3$ & $3.0 \pm 6.5$ & 0.265 & $5.3 \pm 5.8$ & $4.9 \pm 4.5$ & 0.091 \\
\hline
\end{tabular}

Values are presented as number or mean \pm standard deviation, unless otherwise stated. Bold type is considered statistically significant.

izations and readmission include bladder dysfunction, epilepsy, and chronic respiratory insufficiency [20]. Although our numbers do not support this completely, neurogenic bladder is also very common and predisposes to renal damage [23]. Wounds and dressings get easily contaminated with colonized urine, which increases the risk of infection and prevents the wound from healing. In our study, $35 \%$ of patients with myelomeningocele selfcatheterized, and $54 \%$ had vesicoureteral reflux with UTI in the preoperative evaluation [23].
Two patients presented with seizures in the immediate postoperative period, with one patient having a past history of Rett syndrome. Interestingly, this increased the LOS in the ICU [24]. Hence, we recommend checking anticonvulsant plasmatic levels in advance.

A good preoperative evaluation and preparation is mandatory in patients with respiratory failure undergoing surgery. Patients who present with inadequate ventilatory mechanics due to hypotonicity, spasticity, or trunk shortening have a high incidence of pneumonia during 
their lives at a rate of 8.0 for every 100 patients $[25,26]$. Importantly, our study findings support that the number of LRTIs in patients decreased to $51.9 \%$ after surgery. This decrease is significant as it is associated with important increases in the overall quality of life and life expectancy.

To expand more on the improvement of quality of life after surgery, our results disclosed significant decreases in the incidences of UTI, LRTI, and epilepsy after the surgery. These decreases relieve patients and parents of significant non-scoliosis-related issues; a stable spine allows for better hygiene, easier access to catheterization (leading to a decreased risk of developing UTIs), better ventilation and less gastroesophageal reflux, and therefore less incidence of LRTIs. We can only theorize regarding the significant decrease in seizures, but a vicious position is painful and stressful. We hypothesize that the relief caused by surgery probably diminishes the reactive threshold to trigger a seizure.

Cardiac sequelae stemming from neuromuscular pathologies include cardiac malfunctions due to primary myocardiac alterations or collagen quality. High-magnitude curves are associated with increased deterioration of diastolic function. Due to the curve magnitude, the cardiac evaluation oftentimes may be incomplete. In one study, only $49.3 \%$ of patients were able to undergo an appropriate echocardiogram, with 33\% of them undergoing surgery suffering from a minor cardiac-related complication after surgery [27]. In our patient series, $100 \%$ of them underwent an echocardiogram, but ejection fraction (EF) could be measured in only $23 \%$ of them. We did not find any correlation between the curve magnitude/rotation and the ability to measure the EF. Importantly, we found that curves $>80^{\circ}$ were associated with an $\mathrm{EF}<60 \%$, thus providing further evidence of the importance of preventing neuromuscular patients from reaching such levels of deformity.

Myocardial stress correlates with poor nutrition, and poor nutrition has been shown to correlate with complications, poor surgical results, and greater length of hospital stay [28]. Due to hypotonia or spasticity, deglutition capacity is diminished and therefore serves as a significant obstacle to adequate weight gain [15]. Furthermore, we found that a lower preoperative glycemia is a predictor of complications, even if it is not in hypoglycemia levels. This point must be considered when scheduling surgeries as patients generally fast for long hours and reach the surgery at low glycemic levels. Conversely, obesity has also been correlated with increased risk for complications in the literature. In our country, $60 \%$ of children are overweight or obese, which correlates with a higher complication rate, poor healing, and high levels of inflammatory mediators [28].

Nonambulatory patients have been reported to have a higher prevalence of postoperative complications [11]. We did not find this association in our study; however, all patients with myelomeningocele who had early complications were nonambulatory, whereas those with late complications were ambulatory. Potentially, mechanical loading is more important in ambulatory patients, and immobility predisposes nonambulatory patients to early complications.

Preoperative curve magnitude increased the risk for complications. Fusion to the pelvis, a higher number of levels, and a longer surgical time are associated with longer lengths of hospital stay [11,24]. Although we did not find an association between curve magnitude or rigidity and an increased risk for complications, we believe that this principle holds true, as rigid, bigger curves tend toward longer surgical times and more bleeding, and our study demonstrated that surgical time is a predictor of complications.

Poor or excessive reduction increases the risk for major complications. We found that excessive correction of kyphosis was a predictor of major complications and that unbalanced patients with higher T9SPI values were also at risk. We also found that rigid all-screw constructs predicted the presence of mechanical complications. These patients are typically osteoporotic, and reductions must be adequate and not perfect, as too much expectation may compromise the result of the surgery.

Although in this patient series osteotomies and detethering were not associated with the development of complications, Cognetti et al. [6] reported the opposite result. Considering that $<20 \%$ of the osteotomies included more than one column in our series, we believe that osteotomies increase the surgical time and bleeding and therefore correlate with a major complication-driven event. Detethering during the same surgical time does not add comorbidity; in a study performed on 3,682 pediatric patients who were detethered, there was no increase in the incidence of complications compared with patients undergoing scoliosis surgery without this added procedure [29].

Patients with neuromuscular scoliosis demonstrated 
increased blood loss compared with their adolescent idiopathic scoliosis counterparts [30]. We found that the use of deep drains served as a protective factor for these patients. Therefore, we specifically recommend the use of deep drains in patients with myelomeningocele where dead space after closure remains an issue.

There were some uncommon complications in our patient series, which included small pox in a hospitalized, vaccinated patient, thereby demonstrating how immunosuppressed these patients are after such a major surgery. One patient also suffered a tongue laceration due to malpositioned buccal protection. This finding reveals that we must always be prepared for the unexpected.

Although quality of life measures were not a primary outcome for these children, a decrease in the rate of infections, better ability to eat, better ventilatory parameters, less pain, and a stable spine all make us consider surgery as an efficient method to improve the lives of both children and caretakers, despite the risk of complications.

Based on our study findings, we state that there are important factors that must be considered to reduce the probability of developing complications in patients with neuromuscular scoliosis. In the preoperative setting, obtaining a global patient history and developing a good preoperative plan that considers reduction in terms of established thresholds are key. Intraoperatively, achieving an adequate kyphosis with a balanced center of mass and a low PO, while at the same time decreasing the surgical time and blood loss, can further reduce the probability of developing complications.

\section{Conclusions}

Complications in neuromuscular scoliosis are increased by perinatal comorbidities, long surgical times, and inadequate correction. Appropriate patient preparation and surgical planning are the preventive measures to decrease these complications.

\section{Conflict of Interest}

No potential conflict of interest relevant to this article was reported.

\section{References}

1. Halawi MJ, Lark RK, Fitch RD. Neuromuscular sco- liosis: current concepts. Orthopedics 2015;38:e452-6.

2. Toll BJ, Samdani AF, Janjua MB, Gandhi S, Pahys JM, Hwang SW. Perioperative complications and risk factors in neuromuscular scoliosis surgery. J Neurosurg Pediatr 2018;22:207-13.

3. Jalanko T, Helenius I, Pakarinen M, Koivusalo A. Gastrointestinal complications after surgical correction of neuromuscular scoliosis: a retrospective cohort study. Scand J Surg 2018;107:252-9.

4. Matsumoto M, Miyagi M, Saito W, et al. Perioperative complications in posterior spinal fusion surgery for neuromuscular scoliosis. Spine Surg Relat Res 2018;2:278-82.

5. Turturro F, Montanaro A, Calderaro C, Labianca L, Di Sanzo V, Ferretti A. Rate of complications due to neuromuscular scoliosis spine surgery in a 30-years consecutive series. Eur Spine J 2017;26(Suppl 4):53945.

6. Cognetti D, Keeny HM, Samdani AF, et al. Neuromuscular scoliosis complication rates from 2004 to 2015: a report from the Scoliosis Research Society Morbidity and Mortality database. Neurosurg Focus 2017;43:E10.

7. Rumalla K, Yarbrough CK, Pugely AJ, Koester L, Dorward IG. Spinal fusion for pediatric neuromuscular scoliosis: national trends, complications, and inhospital outcomes. J Neurosurg Spine 2016;25:500-8.

8. Duckworth AD, Mitchell MJ, Tsirikos AI. Incidence and risk factors for post-operative complications after scoliosis surgery in patients with Duchenne muscular dystrophy: a comparison with other neuromuscular conditions. Bone Joint J 2014;96-B:943-9.

9. Sharma S, Wu C, Andersen T, Wang Y, Hansen ES, Bunger CE. Prevalence of complications in neuromuscular scoliosis surgery: a literature meta-analysis from the past 15 years. Eur Spine J 2013;22:1230-49.

10. Lonstein JE, Koop SE, Novachek TF, Perra JH. Results and complications after spinal fusion for neuromuscular scoliosis in cerebral palsy and static encephalopathy using luque galveston instrumentation: experience in 93 patients. Spine (Phila Pa 1976) 2012;37:583-91.

11. Master DL, Son-Hing JP, Poe-Kochert C, Armstrong DG, Thompson GH. Risk factors for major complications after surgery for neuromuscular scoliosis. Spine (Phila Pa 1976) 2011;36:564-71.

12. Kang GR, Suh SW, Lee IO. Preoperative predictors of postoperative pulmonary complications in neuro- 
muscular scoliosis. J Orthop Sci 2011;16:139-47.

13. Modi HN, Suh SW, Hong JY, Cho JW, Park JH, Yang JH. Treatment and complications in flaccid neuromuscular scoliosis (Duchenne muscular dystrophy and spinal muscular atrophy) with posterioronly pedicle screw instrumentation. Eur Spine J 2010;19:384-93.

14. Mohamad F, Parent S, Pawelek J, et al. Perioperative complications after surgical correction in neuromuscular scoliosis. J Pediatr Orthop 2007;27:392-7.

15. Ames CP, Blondel B, Scheer JK, et al. Cervical radiographical alignment: comprehensive assessment techniques and potential importance in cervical myelopathy. Spine (Phila Pa 1976) 2013;38(22 Suppl 1):S149-60.

16. Edwards M. The Barker hypothesis. In: Preedy V, Patel VB, editors. Handbook of famine, starvation, and nutrient deprivation. Cham: Springer International Publishing; 2017. p. 191-211.

17. Hurwitz EE, Simon M, Vinta SR, et al. Adding examples to the ASA-physical status classification improves correct assignment to patients. Anesthesiology 2017;126:614-22.

18. O’Brien MF, Kuklo TR, Blanke KM, Lenke LG; Spinal Deformity Study Group. Radiographic measuremnt manual [Internet]. Milwaukee (WI): Scoliosis Research Society; 2008 [cited 2020 Aug 10]. Available from: https://www.oref.org/docs/default-source/ default-document-library/sdsg-radiographic-measuremnt-manual.pdf?sfvrsn=2\&sfvrsn=2.

19. Mangone M, Raimondi P, Paoloni M, et al. Vertebral rotation in adolescent idiopathic scoliosis calculated by radiograph and back surface analysis-based methods: correlation between the Raimondi method and rasterstereography. Eur Spine J 2013;22:367-71.

20. Hacquebord JH, Leopold SS. In brief: the Risser classification: a classic tool for the clinician treating adolescent idiopathic scoliosis. Clin Orthop Relat Res 2012;470:2335-8.
21. Schwab F, Blondel B, Chay E, et al. The comprehensive anatomical spinal osteotomy classification. Neurosurgery 2014;74:112-20.

22. Berry JG, Glotzbecker M, Rodean J, Leahy I, Hall M, Ferrari L. Comorbidities and complications of spinal fusion for scoliosis. Pediatrics 2017;139:e20162574.

23. Ghorbanpoor M, Kajbafzadeh AM, Nejat F, Rahimi $S$. The incidence of upper urinary tract deterioration among Iranian neonates with spinal dysraphism. Iran J Pediatr 2018;28:e59680.

24. Basques BA, Chung SH, Lukasiewicz AM, et al. Predicting short-term morbidity in patients undergoing posterior spinal fusion for neuromuscular scoliosis. Spine (Phila Pa 1976) 2015;40:1910-7.

25. Campbell RM Jr, Smith MD, Mayes TC, et al. The characteristics of thoracic insufficiency syndrome associated with fused ribs and congenital scoliosis. J Bone Joint Surg Am 2003;85:399-408.

26. Keskinen $H$, Lukkarinen $H$, Korhonen K, Jalanko T, Koivusalo A, Helenius I. The lifetime risk of pneumonia in patients with neuromuscular scoliosis at a mean age of 21 years: the role of spinal deformity surgery. J Child Orthop 2015;9:357-64.

27. Saito $\mathrm{W}$, Inoue $\mathrm{G}$, Imura $\mathrm{T}$, et al. The relationship between preoperative echocardiographic evaluation and spinal deformity in patients with neuromuscular scoliosis. Spine Surg Relat Res 2018;2:48-52.

28. Canada NL, Mullins L, Pearo B, Spoede E. Optimizing perioperative nutrition in pediatric populations. Nutr Clin Pract 2016;31:49-58.

29. Bhimani AD, Selner AN, Patel JB, et al. Pediatric tethered cord release: an epidemiological and postoperative complication analysis. J Spine Surg 2019;5:337-50.

30. Patel SB, Griffiths-Jones W, Jones CS, et al. The current state of the evidence for the use of drains in spinal surgery: systematic review. Eur Spine J 2017;26:2729-38. 